\title{
Flor do Cardo versus Quimosina no Fabrico de Queijos Artesanais
}

\author{
EUCLIDES PIRES*, CARLOS FARO*, ISABEL MACEDO*, CRISTINA ESTEVES*, JOSÉ MORGADO*, \\ PAULA VERÍSSIMO*, DIAS PEREIRA**, DAVID GOMES**
}

\section{Neste trabalho as enzimas coagulantes existentes nas flores dos cardos Cynara cardunculus L. e Cynara humilis $\mathrm{L}$. \\ A DESTABILIZAÇ̃̃̃O DAS MICELAS DE CASEÍNA: PRIMEIRO PASSO NO FABRICO DO QUEIJO} foram caracterizadas em termos de especificidade e de parâmetros cinéticos. 0 s resultados obtidos mostraram que a Cynara cardunculus $\mathrm{L}$. contem duas proteases, uma semelhante à quimosina $\mathrm{e}$ outra semelhante à pepsina, enquanto que a Cynara humilis $\mathrm{L}$ contem apenas 0 componente semelhante à quimosina. Os baixos rendimentos e os sabores amargos referidos por Sá e Barbosa em queijo de vaca fabricados com a flor do cardo são, em parte, explicados neste trabalho por análise da actuação das enzimas daquela flor sobre as caseínas bovinas isoladas. A hipótese da preparação de coalhos com características específicas para um dado tipo de queijo por mistura de extractos das flores dos dois tipos de cardos é avançada.
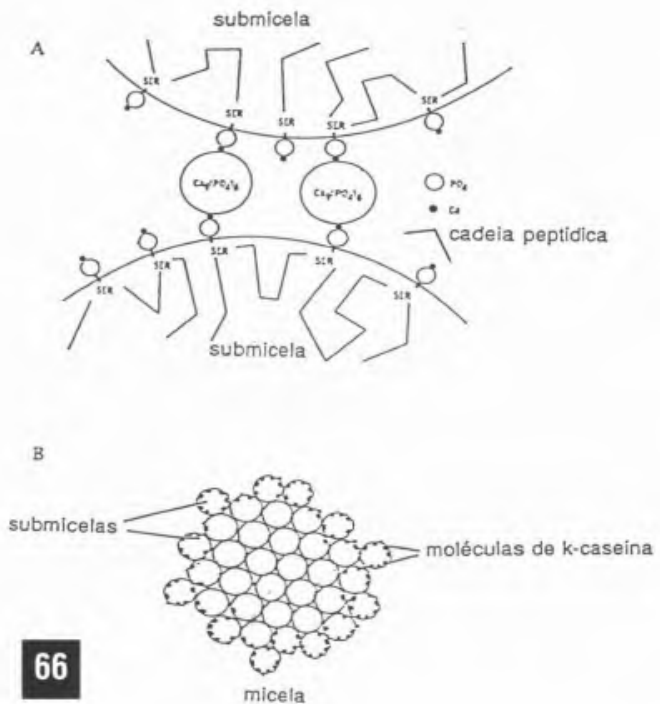

Fig. 1 - Representação esquemática das micelas da caseina. A - Aspecto molecular da ligação entre as submicelas. B-Localizaçâo peritérica da K-caseina (adaptado de Schmidt, 1982)
0 fabrico do queijo inicia-se por um processo de destabilização das micelas de caseina a que se segue a formação de uma rede proteica. 0 soro é então removido desta estrutura, em parte por um processo de contracção espontânea (sinérese) e por pressão exterior. No final desta fase 0 queijo apresenta já uma concentração de proteína 4-5 vezes superior à do leite.

De acordo com o modelo mais aceite, as micelas de caseina são constituídas por submicelas esféricas, com massa de $5 \times 10^{6} \mathrm{Da}$, ligadas entre si por pontes de fosfato de cálcio coloidal (Fig. 1A). Na parte central das submicelas encontram-se as caseinas $\alpha s_{1}, \alpha s_{2}$ e $\beta$ associadas por interacções hidrofóbicas. A k-caseína dispõe-se à superfície das micelas com 0 segmento terminal amínico, mais hidrófobo, interagindo com a parte central da submicela e o segmento do C-terminal, mais hidrófilo, interagindo com o meio aquoso circundante. As submicelas com menor quantidade de k-caseina ocupam posições mais internas na micela enquanto que as com maior quantidade de $\mathrm{k}$ caseina se situam na periferia (Fig. 1B). Para a estabilização desta estrutura contribuem a carga de superfície (potencial Zeta de -15 a $-20 \mathrm{mV}$ ) 0 efeito estérico da protuberância do segmento hidrofílico da k caseína, a presença de resíduos glicosilados e a hidratação.

A destabilização das micelas pode ser promovida por factores físico-químicos (abaixamento do $\mathrm{pH}$ e aumento da temperatura) ou por enzimas (proteases). 0 processo enzimático é, no entanto, o mais utilizado na primeira fase do fabrico do queijo. Neste processo a ligação Phe105Met106 da k-caseína é hidrolisada 0 que leva à libertação da parte mais hidrofílica da k-caseina. A remoção deste segmento facilita a interacção e consequente agregação das micelas.
Uma boa enzima para o fabrico do queijo caracteriza-se por um alto poder coagulante e uma baixa actividade de proteólise geral; isto é a enzima deverá atacar rapidamente a ligação Phe105-Met106 da k-caseína mas ter pouca actividade proteolítica em relacção às outras caseinas. De facto uma proteólise excessiva das caseínas conduz a um baixo rendimento e ou ao aparecimento de sabores amargos.

0 coagulante universalmente utilizado no fabrico do queijo é obtido a partir de extractos do quarto compartimento do estômago de ruminantes jovens (coaIheira) e contém essencialmente quimosina e alguma pepsina. Neste trabalho compara-se as enzimas existentes nas flores de duas espécies de cardo, tradicionalmente utilizadas em Portugal na manufactura de queijos artesanais, com as enzimas existentes nos extractos de estômago de ruminantes jovens.

\section{OBSERVAÇ̃̃ES DE VIEIRA DE SÁ E MANUELA BARBOSA SOBRE A UTILIZAÇÃO GENERALIZADA DOS EXTRACTOS DA FLOR DO CARDO NO FABRICO DO QUEIJO}

Em 1972, num trabalho que se considera uma referência obrigatória nesta área, Sá e Barbosa estudaram a proteólise geral e as propriedades reológicas de queijos produzidos com extractos da flor do cardo e diferentes tipos de leite. Neste trabalho os autores mostraram que os extractos de cardo tinham, no que se refere a leite de vaca, uma actividade proteolitica geral mais elevada 0 que determinava um rendimento mais baixo, além disso, notaram 0 aparecimento de sabores amargos. Apesar destes inconvenientes, os autores concluiram que os extractos de cardo produzem excelentes queijos de ovelha e podem ser satisfatoriamente utilizados como substitutos de coalhos animais no fabrico de queijo de vaca de pasta mole. Naquele trabalho os autores avançaram ainda a hipótese de que a protease da flor do cardo hidrolizaria a k-caseína em posição diferente das outras enzimas coa- 
gulantes. Na sequência destas observações e com vista à identificação dos locais das caseinas susceptiveis à clivagem pela protease da flor do cardo, iniciamos um estudo sistemático da acção desta protease sobre as caseinas isoladas de leite de vaca. Na fase subsequente que se encontra em curso no nosso laboratório, este estudo foi estendido às caseínas isoladas de leite de ovelha $e$ às caseínas nos leites intactos de vaca e ovelha.

\section{ACÇ̃̃O DAS PROTEASES DA FLOR DO CARDO SOBRE AS CASEINAS BOVINAS $\mathbf{k}, \alpha \mathbf{s}_{1}$ e $\alpha \mathbf{s}_{2}$ e $\beta$}

Amostras de caseinas bovinas isoladas e purificadas foram incubadas a $\mathrm{pH}$ 6,2-6,4 com extractos da flor do cardo preparados conforme 0 descrito por Faro (1991). Os produtos de digestão obtidos foram separados por HPLC, a sequência dos terminais amínicos determinada e os peptidos identificados por comparação das sequências com as estruturas primárias das caseinas. Para o caso da k-caseina concluíu-se que a ligação clivada é a Phe105-Met106, tal como sucede para 0 caso das outras enzimas coagulantes (Faro et al., 1992, Macedo et al.,1993). Estudos da cinética da hidrólise desta ligação mostram que 0 coeficiente proteolítico da enzima é da mesma ordem de grandeza mas que $0 \mathrm{Km}$ é mais baixo do que 0 determinado para outras enzimas coagulantes. Em conclusão poderá dizer-se que as diferenças obtidas nos queijos preparados com esta enzima em relação a outras enzimas, não

Fig. 2 - Comparação da hidrólise das caseínas $\alpha$ e $\beta$ pela protease de Mucor miehei (M-digestão de 10 e 45 min.)

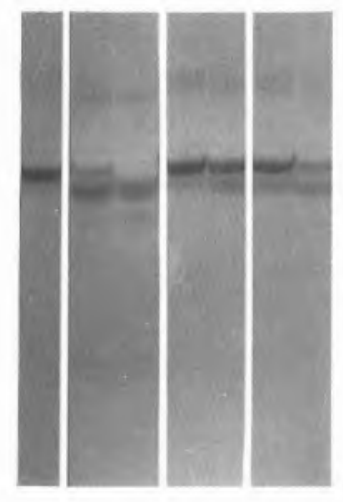

Controlo C R M estão relacionadas com a acção sobre a k-caseina.

No que se refere às caseinas $\alpha$ s1, $\alpha$ s2 e $\beta$ a situação é bastante diferente. Efectivamente, a flor do cardo mostrou-se capaz de clivar mais ligações nestas caseinas do que enzimas coagulantes comuns, Fig.2 (Morgado 1990) e Tabela I (Macedo, 1993).

Os resultados obtidos por Morgado (1990) e Macedo (1993) permitem avançar algumas explicações para 0 aparecimento de sabores amargos e baixos rendimentos referidos por Sá e Barbosa (1972) em queijos de vaca produzidos com a protease da flor do cardo. De facto, os sabores amargos em queijos são atribuidos em parte a peptidos hidrofóbos de pequena dimensão ( $Q$ superior a 1400 $\mathrm{cal} /$ mole e $\mathrm{MM}<6000 \mathrm{Da}$ ) em geral provenientes da $\beta$-caseína. Os dados apresentados na Fig.2 e na Tabela I mostram que a protease da flor do cardo, para além de atacar a $\beta$-caseína, actua sobre a região volumosa e hidrofóbica, Ala163-Val167 da $\alpha s_{1}$ caseína hidrolizando todas as ligações peptídicas deste segmento produzindo peptidos potencialmente amargos. Por sua vez a $\alpha$ s2 caseina, normalmente considerada resistente ao ataque por proteases ácidas, é também hidrolizada pela protease da flor do cardo com produção de um peptido amargo. Curiosamente este peptído tem caracteristicas comuns a neuropeptídos com actividade opioide. Finalmente, também a $\beta$-caseina, é mais atacada pela protease da flor do cardo do que pela quimosina na região Ala189-Tyr193 com a produção de vários peptidos, quatro dos quais potencialmente amarges.

No que se refere ao rendimento, este é necessariamente afectado pelo ata-

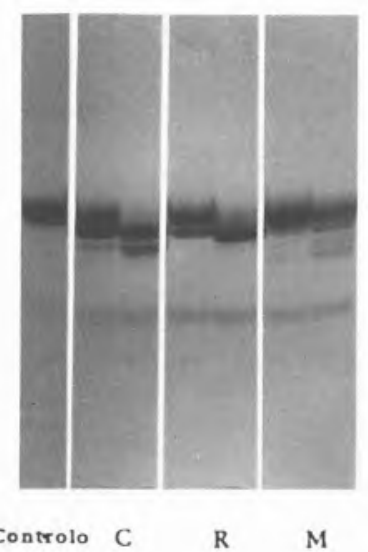

B-caseína
TABELA I. COMPARAÇÃO DAS LIGAÇÕES HIDROLIZADAS PELA QUIMOSINA E PELA PROTEASE DA FLOR DO CARDO NAS CASEINAS BOVINAS ISOLADAS.

\begin{tabular}{llll}
\hline $\begin{array}{l}\text { Caseinas / } \\
\text { /Enzimas }\end{array}$ & $\alpha$ s1 & $\alpha$ s2 & $\beta$ \\
\hline Quimosina & Phe23-Phe24 & & \\
\hline Protease & Phe23-Phe24 & Ala189-Tyr190 \\
Leu192-Tyr193 \\
da flor & Phe145-Tyr146 & & \\
do cardo & Leu149-Phe150 & Leu127Thr128 \\
& Phe153-Tyr154 & & Leu165Ser166 \\
& Leu156-Asp157 & Phe88-Tyr89 & Ala189-Phe190 \\
& Ala163-Trp164 & Tyr95-Leu96 & Phe190Leu191 \\
& Trp164-Tyr165 & & Leu191Leu192 \\
& Tyr165-Tyr166 & Leu192-Tyr193 \\
& Tyr166-Val167 & & \\
\hline
\end{tabular}

que mais extensivo às caseínas $\alpha \mathrm{s}_{1}$, $\alpha s 2$ e $\beta$ pela protease da flor do cardo, produzindo-se eventualmente peptidos que são arrastados com o soro durante a sinérese e prensagem do queijo. Em particular a remoção de parte do segmento do terminal carboxilico da $\beta$ caseína terá efeitos adversos na formação do coágulo e na sinérese uma vez que aquele segmento parece desempenhar um papel importante na estabilização dos polimeros que a $\beta$-caseína forma com a $\alpha$ s 1 -caseina.

Os resultados acima referidos indicam à partida que a protease da flor do cardo ataca com maior facilidade regiões volumosas e hidrofóbicas das caseínas isoladas do que a quimosina. Deve no entanto chamar-se a atenção para que actuação no leite intacto pode não ter necessariamente a mesma extensão já que a associação das caseínas em micelas poderá tornar inacessiveis ao ataque algumas daquelas regiões.

\section{CONTÉUDO QUALITATIVO \\ DAS PROTEASES DOS CARDOS MAIS UTILIZADOS NO FABRICO DO QUEIJO}

No fabrico dos queijos artesanais utilizam-se com mais frequência duas espécies distintas de cardos - Cynara cardunculus L. e Cynara humilis L.. A Cynara cardunculus L., que apresenta um porte maior, existe em menor abundância, estando cada vez mais restringi- 

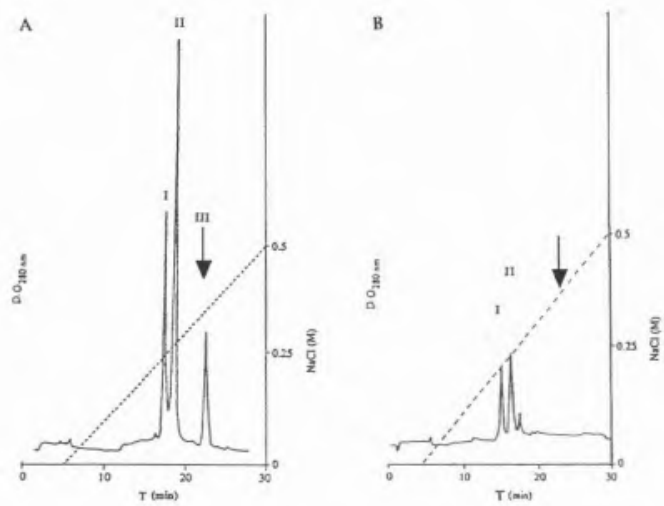

Figura 3 - Cromatografia em Mono $Q$ das proteases de: A - Cynara cardunculus L. B - Cynara humilis L.

da a zonas onde the é dedicado algum cuidado. A Cynara humilis L., por seu lado, tem um porte menor e cresce espontânea e abundantemente na Zona Centro e no Alentejo. Tradicionalmente na Beira Alta, considera-se a Cynara cardunculus $\mathrm{L}$ como 0 cardo ideal para 0 fabrico do queijo da Serra, sendo mesmo cultivado por alguns produtores. Análise da flor do cardo vendida em establecimentos da Guarda revelou no entanto que uma boa parte da flor do cardo ali vendida é de Cynara humilis L..., proveniente do Alentejo.

Isolamento e purificação das proteases das flores de Cynara cardunculus L e de Cynara humilis L., mostrou que 0 conteúdo qualitativo das enzimas presentes nestas duas espécies é diferente (Fig. 3). Face a este resultado passouse à caracterização destas formas em termos de especificidade e de parâmetros cinéticos. A especificidade das formas isoladas foi investigada usando a cadeia $\beta$ oxidase da insulina (Verissimo, 1994). Para 0 efeito, cada forma foi incubada com o substrato a pH 3 por 3horas. Os fragmentos da insulina obtidos foram separados por RP-HPLC e a sequência do terminal amínico determinada por sequenciação automática. Os resultados obtidos mostram que as formas indicadas com I e II hidrolizam as ligações Leu15-Tyr16, Leu17-Val18 e Phe25-Tyr26. Por sua vez a forma III hidroliza as ligacinéticos $\mathrm{Km}$. Kcat e $\mathrm{Km} / \mathrm{Kcat}$ foram também investigados utilizando como substrato o peptido cromofórico Leu-Ser-Phe $\left(\mathrm{NO}_{2}\right)-N$ eu-Ala-Leu-OMe (Tabela II). Como conclusão deste

TABELA II. COMPARAÇÃO DOS PARÂMETROS CINÉTICOS DAS PROTEASES DE CYNARA CARDUNCULUS L. COM OS DA PEPSINA E QUIMOSINA BOVINA

\begin{tabular}{llll}
\hline Enzimas & $\boldsymbol{K m}\left(\mathbf{m M}^{-1}\right)$ & $\boldsymbol{K c a t}\left(\mathbf{s}^{-1}\right)$ & $\boldsymbol{K c a t} / \boldsymbol{K m}\left(\mathbf{m M}^{-1} \mathbf{s} \mathbf{s}^{-1}\right)$ \\
\hline Cardosina A & 1.08 & 23.6 & 21.87 \\
Quimosina & 0.98 & 25.1 & 25.6 \\
Cardosina B & 0.08 & 85.5 & 1057 \\
Pepsina & 0.03 & 54 & 1640 \\
\hline
\end{tabular}

estudo pode dizer-se que, em termos de especificidade e de parâmetros cinéticos as formas I e II são semelhantes entre si e semelhantes à quimosina $e$ que a forma III é semelhante à pepsina. Pode ainda concluir-se que a Cynara cardunculus L. possui uma componente semelhante à quimosina e outra semelhante à pepsina enquanto que a Cynara humilis L. contém apenas um componente semelhante à quimosina.

A designação vulgar para estas proteases, proposta e aceite pela maioria dos cientistas presentes no $5^{\circ}$ Congresso Internacional das Proteases Aspárticas realizado em Setembro de 1993 no Japão, foi a de Cardosin (do radical cardo $+\sin )$ (Faro et al. 1993).

\section{IMPLICAÇÕES DO TIPO DE CARDO UTILIZADO NAS PROPRIEDADES REOLÓGICAS E ORGANOLÉPTICAS DO QUEIJO}

A presença de um componente proteolítico adicional com especificidade diferente em Cynara cardunculus L. levanta a questão de qual 0 efeito deste componente nas propriedades reológicas e organolépticas dos queijos preparados com este cardo em comparação com os preparados com Cynara humilis L..

Um estudo ainda muito preliminar feito com leite de ovelha (Esteves 1994) aponta para a diminuição da dureza e da adesividade, medidas nas fases iniciais, dos queijos produzidos com Cynara cardunculus L. (Tabela III).

0 trabalho descrito nesta comunicação, para além de esclarecer alguns aspectos físico-químicos da actuação da protease da flor do cardo, chama a atenção, pela primeira vez, para as diferenças qualitativas no conteúdo das proteases presentes nas flores dos cardos mais vulgarmente utilizadas no fabrico de queijos artesanais. Tal como já se faz na preparação industrial de coalhos animais, onde se doseiam as proporções de quimosina e pepsina, também no caso do cardo, a mistura de extractos de flores das duas espécies de cardo parece ser um caminho a seguir para a obtenção de coalhos com proporções dos dois componentes adequadas ao tipo de queijo em vista.

\section{* Departamento de Bioquimica da Universidade de Coimbra "Escola Superior Agrária de Coimbra}

\section{REFERÊNCIAS}

1. C. L. Esteves, Tese de Mestrado (em preparação)

2. C. J. Faro, Tese de Doutoramento, Universidade de Coimbra. Coimbra, Portugal, 1991.

3. C. J. Faro, Biotechnol. Lett. 14 (1992) 841.

4. I. Macedo, Tese de Doutoramento, Universidade de Coimbra. Coimbra, Portugal, 1994.

5. I. Macedo, J. Agric. Food Chem. 41 (1993) 1537

6. J. Morgado, Tese de Mestrado, Universidade de Coimbra. Coimbra, Portugal, 1991.

7. V. Sá, M. Barbosa, J. Dairy Res. 39 (1972) 335.

8. D. Schmidt, (Fox, PF ed., Els. Appl. Sci. Publ. New York, vol. 1, 61). Developments in Dairy Chemistry, 1982.

9. P. Veríssimo, Tese de Doutoramento (em preparação)

\section{TABELA III. PARÂMETROS TEXTURAIS DE COALHADAS PRODUZIDAS POR CYNARA CARDUNCULUS L. E POR CYNARA HUMILISL.} Dureza $\left(10^{-3} \mathrm{~N}\right)$ Adesividade $\left(10^{-1} \mathrm{M}^{2}\right)$

\begin{tabular}{lll}
\hline C. cardunculus & 107,8 & 0.4 \\
C. humilis & 166,6 & 0.6 \\
\hline
\end{tabular}




\section{CIÊNCIAS FÍSICO-QUÍMICAS Manuais para o ano lectivo 94/95}

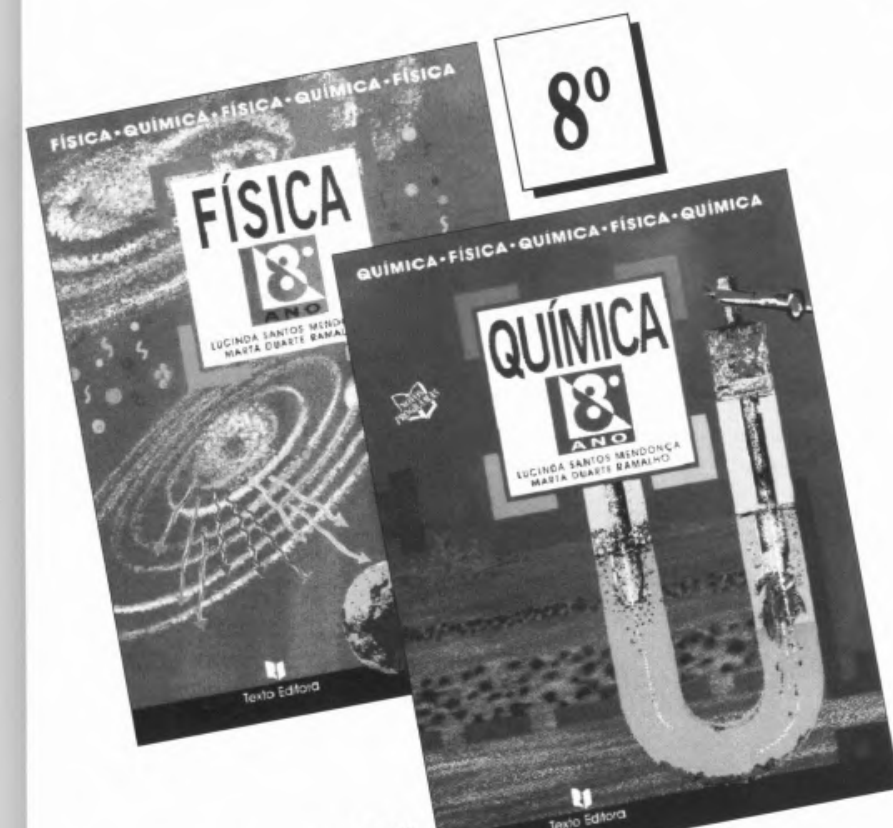

LUCINDA MENDONCCA
MARTA RAMALHO
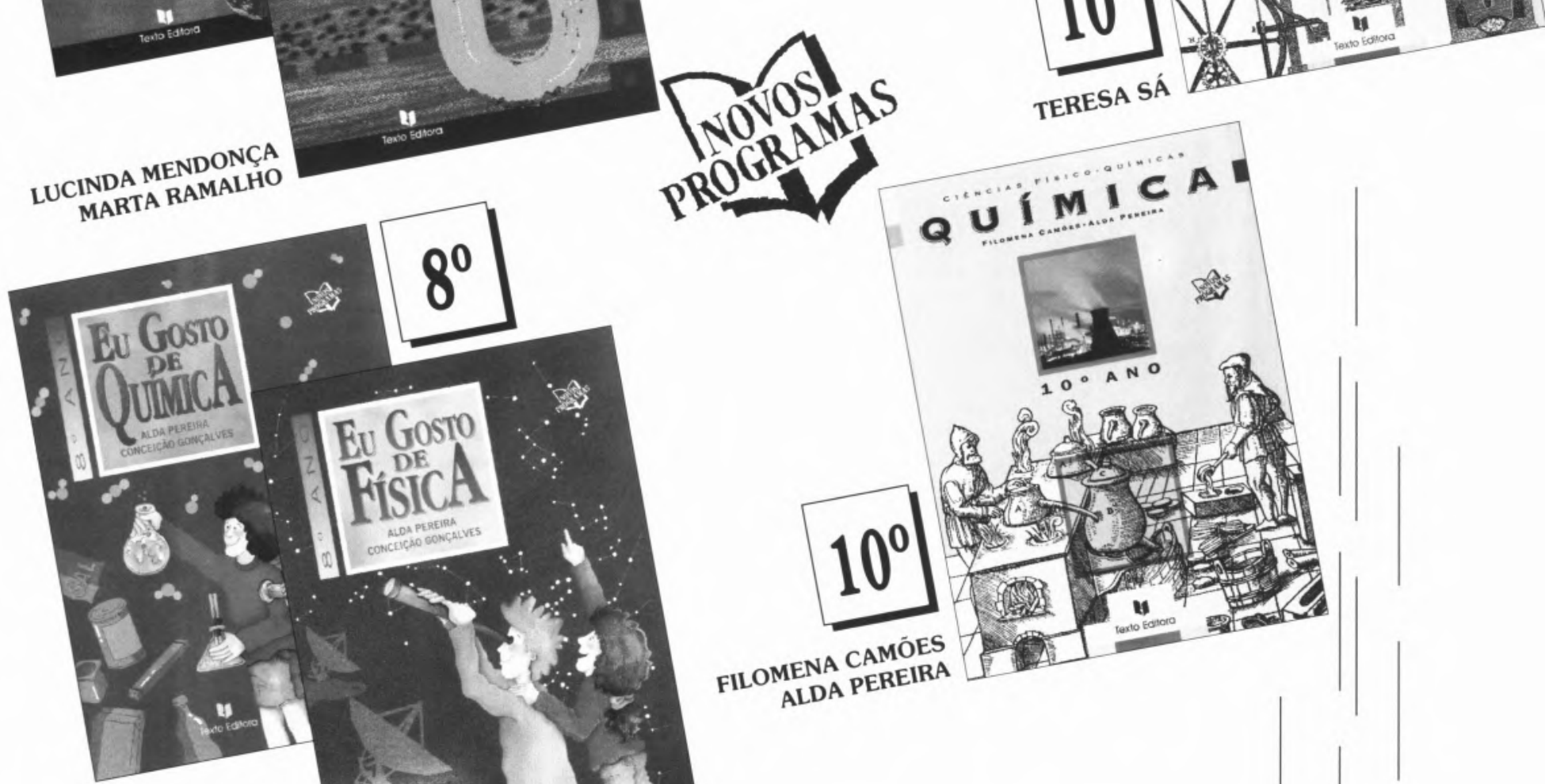

CONCEIÇÁO GLDA PEREIRA
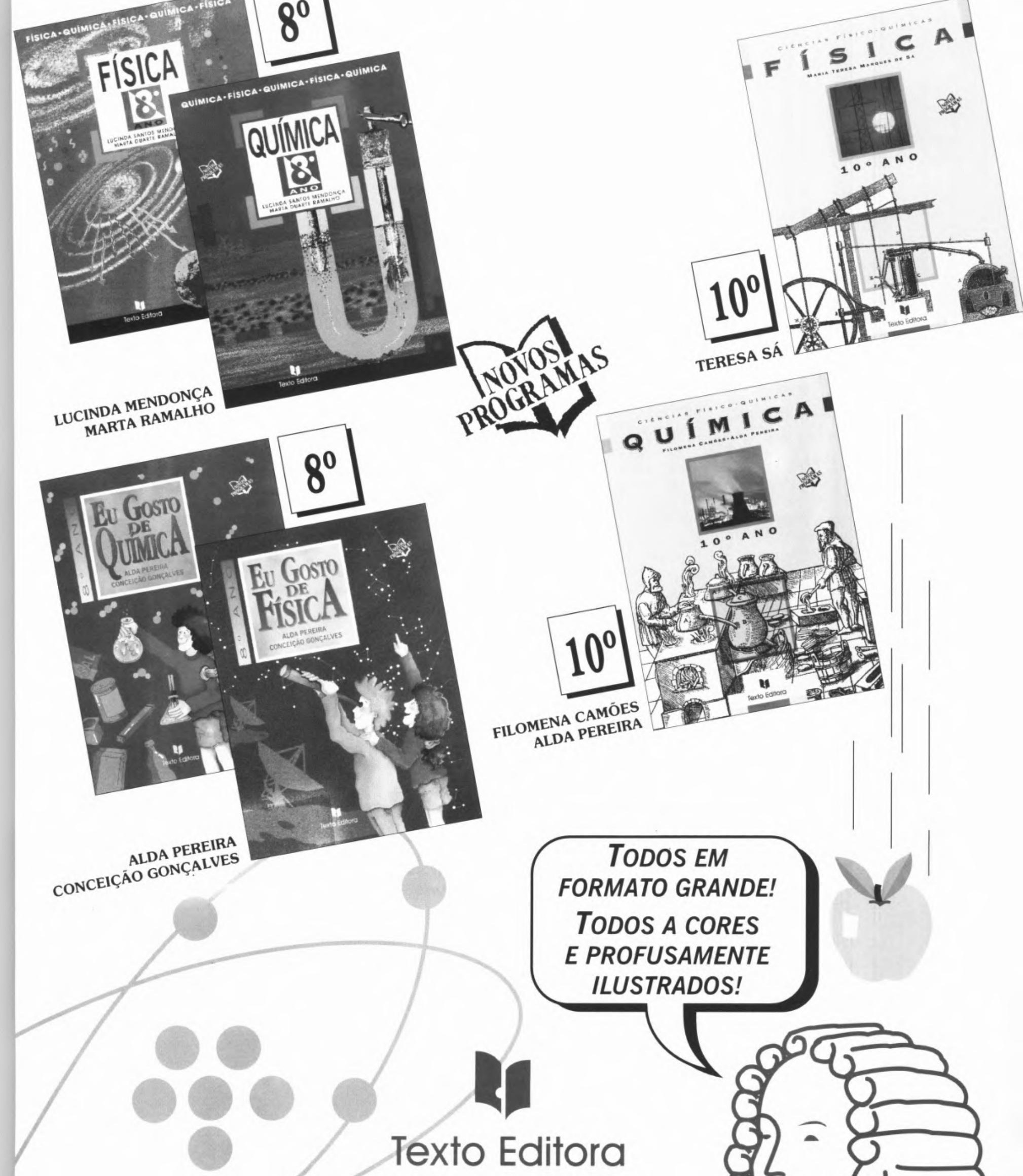